\title{
Relações entre Criatividade, Esperança, Otimismo e Desempenho Profissional
}

\author{
Livia Lopes Lucas Carlomagno ${ }^{1}$ \\ Jean Carlos Natividade \\ Manoela Ziebell de Oliveira \\ Claudio Simon Hutz \\ Laboratório de Mensuração da Universidade Federal do Rio Grande do Sul, \\ Porto Alegre, Rio Grande do Sul, Brasil
}

\begin{abstract}
Resumo
O desempenho profissional nas organizações diz respeito aos comportamentos que são apresentados pelos empregados a fim de atender às expectativas das atividades que realizam. A importância desses comportamentos é evidente para o sucesso da organização e, consequentemente, verifica-se uma grande preocupação em promovê-los. Um dos requisitos para promover o desempenho profissional é conhecer as variáveis relacionadas a ele. Neste estudo buscou-se elucidar as relações entre desempenho profissional e esperança, otimismo e criatividade, bem como testar um modelo preditivo do desempenho a partir dessas variáveis. Participaram 194 funcionários de seis empresas privadas que trabalhavam em funções de vendas e recuperação de crédito. Verificaram-se correlações positivas entre desempenho e esperança, otimismo e criatividade. Quando as variáveis foram tomadas em conjunto a esperança e a criatividade foram aquelas capazes de predizer o desempenho. Esses resultados permitem concluir que o estímulo ao potencial criativo e ao desenvolvimento da esperança dos funcionários é fundamental para obter um desempenho organizacional superior e promover o florescimento dos indivíduos.
\end{abstract}

Palavras-chave: Desempenho no trabalho, esperança, otimismo, criatividade.

\section{Relationships between Creativity, Hope, Optimism and Work Performance}

\begin{abstract}
Job performance in organizations refers to the real behaviors exhibited by employees to achieve the expectations of success of organizations. The relevance of these behaviors for organizational success is evident and, therefore, we observe a great concern in promoting them. One of the requirements to promote job performance is to learn what variables are related to it. This study aimed to elucidate the relationship between job performance, hope, optimism and creativity, and also test a model to predict job performance based on these variables. The participants were 194 employees from six private companies, working in sales and credit recovery functions. The results indicated positive correlations between performance, hope, optimism and creativity. When the variables were taken together hope and creativity were those able to predict performance. The results suggest that stimulating creative potential and the
\end{abstract}

1 Endereço para correspondência: Laboratório de Mensuração, Instituto de Psicologia, Universidade Federal do Rio Grande do Sul, Rua Ramiro Barcelos, 2600, sala 101, Bairro Santa Cecília, Porto Alegre, RS, Brasil 90035003. E-mail: livialucas@yahoo.com.br 
development of hope among employees is essential to reach a superior organizational performance and help individuals to flourish.

Keywords: Job performance, hope, optimism, creativity.

\section{Las Relaciones entre la Creatividad, la Esperanza, el Optimismo y el Desempeño Profesional}

\section{Resumen}

El desempeño profesional en las organizaciones se refiere a los comportamientos reales que son presentados por los empleados con el fin de satisfacer las expectativas de suceso de la organización. La relevancia de estos comportamientos es evidente y por eso existe una gran preocupación con su promoción. Uno de los requisitos para promover el desempeño profesional es conocer las variables que se relacionan a él. Este estudio tuvo como objetivo esclarecer la relación entre el desempeño laboral y la esperanza, el optimismo y la creatividad, así como testar un modelo predictivo del desempeño basado en estas variables. El estudio incluyó 194 empleados de seis empresas privadas, que trabajaban en funciones de ventas y recuperación de crédito. Se observaron correlaciones positivas entre desempeño, esperanza, optimismo, y creatividad. Cuando se tomaron las variables en conjunto, esperanza y creatividad fueron aquellas capaces de predecir el desempeño. Estos resultados sugieren que estimular el potencial creativo y el desarrollo de la esperanza entre los empleados es fundamental para obtener un desempeño organizacional superior y promover el florecimiento de los individuos.

Palabras clave: Rendimiento laboral, esperanza, optimismo, creatividad.

Historicamente, o termo desempenho já foi associado tanto a fatores internos do indivíduo, como o grau de motivação e as habilidades técnicas e comportamentais, quanto a fatores externos ao indivíduo, como as condições do contexto em que atua (Lewis \& Heckman, 2006; Siqueira, 2002). Mais recentemente, há quem o entenda como um padrão de comportamentos reais que são apresentados pelos empregados diante de uma expectativa de sucesso organizacional ( $\mathrm{Si}$ queira, 2002). A literatura aponta que o valor atribuído a esses comportamentos pode variar de acordo com o quanto contribuem para a realização dos objetivos do indivíduo e da organização (Aguinis, Gottfredson, \& Joo, 2012). Independentemente de como definem o desempenho, pode-se dizer que, na prática, as organizações almejam atrair e reter os profissionais que apresentam os mais altos desempenhos.

Em decorrência da busca por profissionais de alto desempenho, diversas empresas comprometem aspectos importantes da gestão de recursos humanos, tais como programas de desenvolvimento de líderes e de equipes (Lewis
\& Heckman, 2006). Apesar desses, um dos aspectos que tende a ser preterido na disputa por profissionais de alto desempenho diz respeito à adoção de modelos e estratégias de gestão de pessoas capazes de favorecer e estimular a liberdade, a criatividade, a flexibilidade, o otimismo e a inovação (Moraes \& Lima, 2009). Embora características positivas como essas venham sendo valorizadas pelas organizações e por pesquisadores há algum tempo (Luthans, 2002), apenas recentemente alguns autores passaram a argumentar e a apresentar evidências das implicações da adoção de uma perspectiva positiva no estudo e na gestão do comportamento organizacional (ver mais em Luthans, 2002).

Como decorrência desse movimento, por volta dos anos 2000, verificou-se a emergência da Psicologia Organizacional Positiva (POP), também chamada de psicologia positiva aplicada ao trabalho, ambiente de trabalho positivo, organização positiva, ou comportamento organizacional positivo (Martin, 2005; Turner, Barling, \& Zacharatos, 2002; Wiegand \& Geller, 2005). Dada a sua origem, não surpreende que os temas 
de interesse da POP e os da Psicologia Positiva se sobreponham e que seja possível, segundo alguns autores, classificar esses temas em um único conjunto de três pilares (Peterson \& Byron, 2008). O primeiro pilar refere-se à experiência subjetiva positiva. O segundo pilar está relacionado a estados positivos. Por fim, o terceiro pilar diz respeito a instituições positivas e tem como função promover os primeiros dois pilares, facilitando o florescimento dos indivíduos (Donaldson \& Ko, 2010). Tendo como base esses três pilares, a POP é definida como: a investigação e a aplicação das forças positivamente orientadas dos recursos humanos com o objetivo de medir, desenvolver e gerir tais forças de forma eficaz, visando à melhoria do desempenho individual $\mathrm{e}$ dos resultados organizacionais (Luthans, 2002).

Três variáveis que podem ser incluídas em dois desses pilares são de interesse do presente estudo em função de sua relação com o desempenho organizacional: a esperança e o otimismo (primeiro pilar) e a criatividade (segundo pilar). A esperança caracteriza-se por um estado emocional positivo direcionado ao futuro, que emerge da interação entre rotas e agenciamento na presença de um objetivo. $\mathrm{O}$ agenciamento representa a capacidade do indivíduo de se motivar para iniciar a busca de um dado objetivo e manter-se no caminho para cumprir essa meta. Já as rotas dizem respeito à capacidade percebida de encontrar caminhos que levem ao objetivo desejado (Snyder \& Lopes, 2005). O otimismo, por sua vez, corresponde a uma tendência estável a enfatizar os aspectos favoráveis das situações, ações e eventos, e de esperar que fatos bons aconteçam, ao invés de eventos ruins, tanto no presente quanto no futuro (Carver \& Scheier, 2005). Essa forma de pensar sobre os acontecimentos futuros proporciona maneiras diferentes de lidar com as adversidades. As pessoas que possuem expectativas positivas tendem a persistir mesmo em face de grandes obstáculos e a encarar suas falhas e fracassos como temporários e não como deficiências pessoais (Luthans \& Youssef, 2004; Wright \& Quick, 2009). Por fim, a criatividade pode ser entendida como a produção daquilo que é novo, útil e valioso para solucionar um problema (Amabile, 1993). Amabile (1993, 1998) de- fende que o processo criativo do indivíduo é uma função de três componentes individuais e contextuais: conhecimentos, habilidades de pensamento criativo e a motivação. Os conhecimentos podem ser técnicos, processuais ou intelectuais, abrangendo tudo aquilo que a pessoa conhece ou sabe fazer no amplo domínio do seu trabalho. As habilidades de pensamento criativo referem-se a como a pessoa lida com problemas e encontra soluções, ou seja, sua capacidade de criar novas combinações para ideias já existentes. Essa capacidade depende tanto da personalidade do indivíduo, como da sua maneira de pensar e trabalhar (Amabile, 1998). Por fim, a motivação pode ser definida como a paixão interna para resolver uma dada questão (Amabile, 1998; Csikszentmihalyi, 1996).

No contexto organizacional, há evidências de que a correlação entre a esperança e o desempenho profissional é positiva e significativa, porém baixa ( $r=0,24$ a $r=0,29$; Luthans, Avolio, Avey, \& Norman, 2007; Peterson \& Byron, 2008). Uma metanálise realizada por Luthans e Youssef (2004) demonstrou que profissionais com altos níveis de esperança tiveram melhores resultados nas avaliações de desempenho feitas pelo supervisor (Luthans \& Youssef, 2004). Em outro estudo, Youssef e Luthans (2007) constataram que a esperança tinha correlação significativa e positiva com as autoavaliações de desempenho $(r=0,22)$ e com o desempenho avaliado por supervisores $(r=0,16)$ e poderia predizer significativamente os resultados de autoavaliações de desempenho $(\beta=0,21)$. Os resultados indicaram ainda que o desempenho poderia ser predito mais adequadamente pela esperança do que pela resiliência. Segundo os autores, tais resultados devem-se ao fato de a esperança ser capaz de promover o aumento da motivação e da confiança (agenciamento), levando os profissionais a construir estratégias para superar desafios no trabalho (rotas). Peterson e Byron (2008) verificaram ainda que profissionais mais esperançosos nas áreas de vendas, corretores de hipotecas e executivos tiveram um desempenho maior do que os menos esperançosos. Na tentativa de entender melhor essa relação os autores mostraram que indivíduos com alta esperança 
apresentaram um leque maior e com mais qualidade de soluções para os problemas do que os seus pares.

Em relação ao otimismo, foram verificadas associações positivas com o desempenho, e verificou-se que pessoas com maiores níveis de otimismo têm níveis mais altos de satisfação e vendem aproximadamente $37 \%$ mais do que as com menores índices de otimismo (Luthans \& Youssef, 2004). Esse resultado é de particular interesse no contexto organizacional, uma vez que evidencia a possibilidade de orientar o indivíduo para que ele atue de forma mais otimista através de intervenções pontuais, como programas de treinamento e desenvolvimento (e.g. Carver \& Scheier, 2005).

Já no que tange à criatividade, verificou-se que a qualidade da relação de troca com o líder de equipe, por exemplo, pode impactar o desempenho criativo do empregado de várias maneiras (e.g. Tierney, Farmer, \& Graen, 1999). Há ainda evidências mostrando que funcionários com alta qualidade de troca na relação com os líderes têm desempenhos superiores aos de seus pares com baixa qualidade de troca, em tarefas mais desafiadoras e importantes (Amabile \& Gryskiewicz, 1988). Por sua vez, a capacidade de engajar-se em tarefas desafiadoras e importantes está relacionada ao desempenho criativo no local de trabalho (Amabile \& Gryskiewicz, 1988). Considerando esses fatores, a criatividade pode ser interpretada como vital para a vantagem competitiva de uma organização, pois quando os colaboradores pensam de maneira criativa, tendem a apresentar ideias e sugestões inovadoras que oferecem à organização a base para o desenvolvimento (Holmes, 2007).

A descrição das variáveis de interesse desse estudo, e das investigações sobre suas relações com o desempenho, remete a dois aspectos importantes da POP. O primeiro deles diz respeito ao interesse por estados passíveis de modificação e não por traços estáveis dos indivíduos. Tal característica evidencia os potenciais benefícios da aplicação da POP para as organizações, uma vez que reconhece a possibilidade de aprender, desenvolver, modificar e gerir as características positivas dos indivíduos no local de trabalho
(Luthans, 2002). O segundo aspecto diz respeito à ênfase no aprimoramento teórico e técnico, visando à mensuração adequada, às investigações empíricas e ao desenvolvimento continuado da área.

Esse segundo ponto pode ser exemplificado pela meta-análise realizada por Donaldson e Ko (2010). Os autores analisaram 172 artigos relacionados à POP publicados em periódicos revisados por pares entre os anos de 2001 e 2009. Os resultados indicaram que o número de publicações empíricas relacionadas à POP aumentou, principalmente, a partir de 2008. Contudo, os autores destacam que apenas $34 \%$ dos artigos analisados derivaram de estudos empíricos. Isso mostra que embora haja um esforço para buscar evidências capazes de confirmar ou refutar afirmações teóricas em POP, esse ainda é um campo em desenvolvimento e como tal carece de estudos empíricos para se estabelecer como uma disciplina sólida. Ainda, de acordo com os autores, $72 \%$ das publicações incluídas na meta-análise foram produzidas nos Estados Unidos e os $28 \%$ restantes tiveram origem em países da África, da Ásia, da Europa e da Oceania. No entanto, nenhum estudo incluído na análise foi desenvolvido na América Central e na América do Sul. Esse resultado reforça a importância de novos estudos relacionados à $\mathrm{POP}$ em países como o Brasil.

Alinhados com as proposições da POP e considerando a configuração atual da área, os autores deste trabalho se propõem a ampliar o escopo de discussões sobre o campo do comportamento organizacional orientado para características positivas, e apresentar dados empíricos capazes de fundamentar ações de desenvolvimento de pessoas nas organizações. Mais especificamente, buscou-se investigar, em uma amostra de profissionais brasileiros, se a esperança, o otimismo e a criatividade estão relacionadas ao desempenho profissional, além de testar o poder preditivo daquelas variáveis em conjunto sobre o desempenho profissional. Para tanto, compreendeu-se desempenho como um conjunto ou padrão de comportamentos reais apresentados pelos empregados diante de uma expectativa com vistas ao sucesso da organização (Siqueira, 2002). 
A escolha das variáveis a serem relacionadas com o desempenho profissional neste estudo buscou contemplar dois dos pilares da POP: as experiências positivas, representadas pela esperança e pelo otimismo, e os estados positivos, representados pela criatividade. Uma vez que não há consenso sobre a melhor forma de operacionalizar e mensurar o nível de positividade das instituições, variáveis relacionadas a esse pilar não foram contempladas nesta pesquisa (para mais detalhes sobre a lacuna na mensuração desse pilar ver Donaldson \& Ko, 2010). Entre as variáveis abarcadas nesta pesquisa, a esperança e otimismo já foram destacadas como preditoras do desempenho profissional (e.g. Avey, Luthans, $\&$ Youssef, 2009). Contudo, essas duas variáveis estão entre aquelas que receberam menos atenção dos pesquisadores da área quando comparadas a conceitos-chave como autoeficácia, bem estar subjetivo e inteligência emocional. A criatividade também tem se mostrado importante para explicar o desempenho nas organizações (e.g. Montag, Maertz, \& Baer, 2012). Além disso, ainda que haja estudos com cada uma das variáveis isoladamente, pouco se conhece sobre o poder preditivo desses construtos conjuntamente sobre o desempenho profissional.

\section{Método}

\section{Participantes}

Participaram 194 profissionais de seis empresas privadas da região metropolitana de Porto Alegre. Dos participantes, $71,6 \%$ eram mulheres, a média de idade foi de 30,0 anos $(D P=11,2)$. A maioria dos participantes, $58,6 \%$, tinha o ensino médio completo, tinham o ensino superior incompleto $20,4 \%$ deles, $12,9 \%$ tinham ensino superior completo e os demais $8,1 \%$ tinham menos que o ensino médio. Todos os participantes desempenhavam funções relacionadas ao atendimento ao público $(56,7 \%$ eram recuperadores de crédito; 33,3\% eram vendedores) em empresas do ramo imobiliário, de telefonia, de cobrança, de consultoria, de logística, e de vestuário. $\mathrm{O}$ tempo dos funcionários nas empresas variou de um a 96 meses $(M=15,7 ; D P=16,7)$.
O salário mensal recebido variou de $\mathrm{R} \$ 360,00$ a $\mathrm{R} \$ 10.000,00(M=\mathrm{R} \$ 1.480 ; D P=\mathrm{R} \$ 1.421)$.

A amostra foi escolhida utilizando o critério de conveniência. Diversas empresas foram contatadas e convidadas a participar do estudo. Foram incluídas na amostra apenas aquelas que consentiram que seus subordinados e respectivos supervisores respondessem a pesquisa. A amostra dos supervisores foi composta por 21 pessoas, nove homens e 12 mulheres, a média de idade deles foi de 32,5 anos $(D P=7,96)$.

\section{Instrumentos}

Utilizaram-se dois questionários autoaplicáveis de respostas fechadas, um destinado aos funcionários das empresas e outro destinado aos seus respectivos supervisores. Em ambos havia questões sociodemográficas como sexo, idade, escolaridade. No questionário destinado aos funcionários havia questões relacionadas ao emprego atual como tempo na empresa, salário recebido, cargo e função desempenhada, além dos seguintes instrumentos: Escala Vazquez-Hutz de Avaliação de Desempenho-EVHAD (Vazquez \& Hutz, 2008); Escala de Esperança Traço (Pacico, Zanon, Bastianello, \& Hutz, 2013); Life Orientation Test-Brazil (Bastianello, Zanon, Pacico, \& Hutz, no prelo); Escala de Estratégias para Criar no Trabalho (Moraes \& Lima, 2009). No questionário dos supervisores havia apenas o EVHAD para que eles respondessem avaliando cada um de seus subordinados.

A Escala Vazquez-Hutz de Avaliação de Desempenho-EVHAD (Vazquez \& Hutz, 2008) tem como objetivo medir o desempenho de funcionários ligados a setores de atendimento ao público e contém sete subescalas com 87 itens no total. A escala pode ser respondida tanto diretamente pelo funcionário quanto pelo seu supervisor. $\mathrm{O}$ instrumento avalia sete dimensões do desempenho relacionadas a competências essenciais à atividade de atendimento ao cliente: habilidades técnicas, manejo do estresse situacional, foco na produtividade, trabalho em equipe, negociação com assertividade, foco no cliente e foco nos resultados. A consistência interna (alfa de Cronbach) varia de 0,87 a 0,97 entre os fato- 
res da escala. Os itens são em formato de afirmativas que representam situações de trabalho. Os respondentes devem indicar, utilizando uma escala de 1 a 5 , a frequência com que cada uma das situações ocorre com eles (ou com o profissional que estão avaliando), tal que 1 significa 'nunca' e 5 significa 'sempre'. Os resultados geram escores para cada uma das dimensões avaliadas e também um escore geral do desempenho. Para fins deste estudo utilizou-se apenas o escore geral de desempenho.

A Escala de Esperança Traço (Pacico et al., 2013) contém oito itens que avaliam a disposição emocional positiva direcionada ao futuro, que emerge da interação entre rotas e agenciamento na presença de um objetivo. Os itens são frases afirmativas para que os participantes respondam o quanto cada uma delas se aplica a eles, em uma escala de cinco pontos, sendo o 1 correspondente a totalmente falso e o 5 a totalmente verdadeiro. $\mathrm{O}$ instrumento apresentou coeficiente alfa superior a $0,80 \mathrm{em}$ seu estudo de elaboração.

O Life Orientation Test - LOT-Brazil (Bastianello et al., 2013) é um teste de autorrelato construído para medir o otimismo disposicional, que pode ser definido em termos de expectativas positivas e negativas com relação a eventos futuros na vida. Consiste em 10 frases afirmativas para que os participantes respondam em uma escala de cinco pontos o quanto concordam com elas, sendo o 1 correspondente a discordo plenamente e o 5 a concordo plenamente. Em seu estudo de elaboração, o instrumento apresentou coeficiente alfa superior a 0,80 .

A Escala de Estratégias para Criar no Trabalho (Moraes \& Lima, 2009) tem como objetivo medir as estratégias usadas para criar no traba1ho. O instrumento acessa os esforços empreendidos pelo indivíduo para favorecer a criação de ideias novas e úteis na resolução de problemas de trabalho. A escala é composta por 44 frases afirmativas para que os participantes julguem em uma escala de 10 pontos a frequência com que elas ocorrem com eles, sendo o ponto 1 correspondente a 'nunca ocorre' e o ponto 10 corresponde a 'sempre ocorre'. O instrumento afere cinco fatores de primeira ordem relacionados ao construto: pensamento flexível, imaginação e introspecção, controle emocional, leitura inspiradora, interação e analogia. Neste estudo utilizou-se o fator Pensamento Flexível. Esse fator refere-se às estratégias cognitivas relacionadas à associação de perspectivas, diversificação de ideias, reformulação de problemas e avaliação extrínseca à geração de ideias e resolução de problemas no trabalho. O fator é composto por 19 itens, divididos em duas dimensões denominadas: (a) Integração e analogia; (b) Soluções e efeitos. No estudo de construção do instrumento, Pensamento Flexivel apresentou coeficiente alfa de 0,95 . Utilizou-se apenas esse fator por se considerar que ele é o que mais se aproxima da conceituação de criatividade de Amabile (1993), uma vez que os demais fatores são relacionados às estratégias comportamentais e de autorregulação.

\section{Procedimentos}

Primeiramente, realizou-se o contato com os diretores das empresas a fim de obter o consentimento para as coletas de dados. A partir disso, foi realizada uma reunião com o departamento de Recursos Humanos e com os gerentes/ supervisores das equipes escolhidas para elaborar um planejamento para a aplicação e entrega dos resultados da pesquisa. Os participantes de cada equipe responderam coletivamente os instrumentos, em cerca de 40 minutos. Os gerentes/supervisores dos funcionários receberam os questionários e foram solicitados a responder sobre seus subordinados e entregar ao pesquisador responsável no prazo de uma semana.

Dois meses após a coleta de dados, foram entregues ao setor de Recursos Humanos, conforme acordo prévio de contrapartida à participação da empresa, os pareceres individuais de cada funcionário que respondeu a pesquisa. As empresas se responsabilizaram por dar o retorno desses resultados aos supervisores e seus subordinados. Por fim, também como contrapartida, foi oferecida às empresas uma palestra sobre a importância do desenvolvimento das características positivas no ambiente organizacional para se atingir os resultados esperados. 


\section{Resultados}

Inicialmente, calcularam-se as médias e desvios-padrões dos instrumentos utilizados. Com relação ao desempenho geral no trabalho, observou-se que nas avaliações realizadas pelos próprios funcionários o desempenho mostrou-se mais elevado $(M=4,27 ; D P=0,37)$ do que na avaliação realizada pelos supervisores $(M=3,67$; $D P=0,58), t(193)=12,3 ; p<0,001 ; d=1,26$. Os demais instrumentos foram respondidos apenas pelos funcionários e apresentaram os seguintes escores: Esperança, $M=4,36 ; D P=0,40$; Otimismo, $M=4,37 ; D P=0,61$; Criatividade-Pensamento Flexível dimensão (a) Integração e analogia, $M=7,37 ; \quad D P=1,32$; Criatividade-Pensamento Flexível dimensão (b) Soluções e efeitos, $M=7,59 ; D P=1,44$.
Para testar as relações entre o desempenho profissional, tanto o autoavaliado quanto o avaliado pelos supervisores, e as variáveis esperança, otimismo e criatividade (pensamento flexível: a-Integração e analogia e b-Soluções e efeitos), foram realizadas correlações de Pearson. Os resultados podem ser vistos na Tabela 1 . Além dessas variáveis, incluíram-se na análise: idade, tempo na empresa e salário mensal recebido pelos participantes. Destacam-se as correlações positivas e moderadas entre desempenho autoavaliado e ambas as dimensões da criatividade, esperança e otimismo. No que diz respeito ao desempenho avaliado pelos supervisores, observa-se correlação positiva, embora fraca, com a dimensão de criatividade Soluções e efeitos.

\section{Tabela 1}

Correlações entre Desempenho Profissional Geral Autoavaliado e Avaliado pelos Supervisores e Criatividade, Esperança e Otimismo

\begin{tabular}{llllllll}
1 & 2 & 3 & 4 & 5 & 6 & 7 & 8 \\
\hline
\end{tabular}

1. Desempenho Autoavaliado

2. Desempenho Avaliação Supervisor

0,03

3. Criatividade-Pensamento Flexível

(a)-Integração e analogia

$0,51 * * \quad 0,01$

4. Criatividade-Pensamento Flexível

(b)-Soluções e efeitos

$0,50 * * \quad 0,17 * \quad 0,73 * *$

5. Esperança

$0,56 * * \quad 0,01 \quad 0,50 * * \quad 0,33 * *$

6. Otimismo

$0,34 * * \quad 0,01 \quad 0,29 * * \quad 0,21 * * \quad 0,45 * *$

7. Idade

$0,19 * * \quad-0,19 * *$

$0,24 * * \quad 0,11$

$0,32 * * \quad 0,22 * *$

8. Tempo no emprego

0,06

0,04

0,10

0,14

0,02

0,07

0,10

9. Salário mensal

$0,15 *$

$-0,11 \quad 0,20 * *$

$0,15^{*}$

$0,19 * * \quad 0,22 * * \quad 0,52 * * \quad 0,10$

Nota. $n=194$ para todas as variáveis, exceto para Esperança: $n=192$ e tempo no emprego: $n=174$. $* p<0,05 ; * * p<0,01$.

Considerando-se as correlações existentes entre as variáveis e delas com o desempenho, testaram-se

quais dentre elas contribuiriam para a explicação da variância do desempenho. Para tanto, delinearam-se dois modelos de regressão linear, um incluindo como variável explicada o desempenho autoavaliado e outro incluindo o desempenho avaliado pelos supervisores. Como variáveis explicativas incluíram-se todas as que apresentaram correlação com desempenho autoavaliado, uma vez que incluir as outras variáveis poderia inflar a variância explicada do modelo com variáveis sem relação com o desempenho. Assim, as variáveis explicativas foram: as dimensões ' $a$ ' e 'b' de Pensamento Flexível-Criatividade; Esperança; Otimismo; idade; salário mensal. Os resultados da análise de regressão, método Enter, podem ser visualizados na Tabela 2.

Tabela 2

Modelos Preditivos do Desempenho Profissional 
Autoavaliado e Avaliado por Supervisores

\begin{tabular}{|c|c|c|c|c|c|c|c|c|}
\hline & \multicolumn{4}{|c|}{ Desempenho autoavaliado } & \multicolumn{4}{|c|}{ Desempenho avaliado supervisores } \\
\hline Variáveis & $\mathrm{b}$ & $\beta$ & $t$ & $p$ & $\mathrm{~b}$ & $\beta$ & $t$ & $p$ \\
\hline Constante & 1,75 & & 7,46 & $<0,001$ & 3,21 & & 6,92 & $<0,001$ \\
\hline $\begin{array}{l}\text { Criatividade-Pensamento Flexível } \\
\text { (a)-Integração e analogia }\end{array}$ & 0,022 & 0,08 & 0,90 & 0,37 & $-0,09$ & $-0,21$ & $-1,81$ & 0,07 \\
\hline $\begin{array}{l}\text { Criatividade-Pensamento Flexível } \\
\text { (b)-Soluções e efeitos }\end{array}$ & 0,08 & 0,30 & 3,65 & $<0,001$ & 0,13 & 0,32 & 3,11 & 0,002 \\
\hline Esperança & 0,35 & 0,38 & 5,49 & $<0,001$ & 0,08 & 0,06 & 0,63 & 0,53 \\
\hline Otimismo & 0,05 & 0,09 & 1,43 & 0,15 & 0,023 & 0,024 & 0,31 & 0,76 \\
\hline Idade & $<0,001$ & $-0,008$ & $-0,12$ & 0,91 & $-0,009$ & $-0,18$ & $-2,15$ & 0,03 \\
\hline Salário mensal & $<0,001$ & $-0,001$ & $-0,01$ & 0,99 & $<0,001$ & $-0,039$ & $-0,47$ & 0,64 \\
\hline$R^{2}$ & & & & & & & 09 & \\
\hline$R^{2}$ ajustado & & & & & & & 06 & \\
\hline$F$ & & 23 , & & & & & $15 *$ & \\
\hline
\end{tabular}

Destacam-se como preditoras significativas do desempenho autoavaliado a dimensão Soluções e

efeitos da criatividade e a Esperança, ambas positivas. Esse modelo explicou $43 \%$ da variância do desempenho autoavaliado. Por outro lado, o modelo explicativo para o desempenho avaliado pelos supervisores explicou $9 \%$ da variância e as seguintes variáveis foram significativas: dimensão Soluções e efeitos da criatividade, positivamente; e idade dos participantes, negativamente.

\section{Discussão}

Os objetivos deste estudo incluíam testar as relações entre desempenho profissional $\mathrm{e}$ esperança, otimismo e criatividade, bem como verificar se aquelas variáveis em conjunto poderiam predizer o desempenho profissional. $\mathrm{O}$ desempenho profissional foi aferido tanto a partir de respostas dos funcionários quanto a partir de respostas dos seus supervisores. Verificou-se discrepância entre essas duas formas de avaliação do desempenho, tal que, em média, a autoavaliação foi superior à avaliação realizada pelos supervisores. Tal discrepância ocorreria porque enquanto os empregados tendem a avaliar o próprio desempenho utilizando os pontos extremos das escalas de avaliação, os supervisores - como representantes das organizações - tenderiam a avaliar o desempenho de seus subordinados de acordo com as normas pré-estabelecidas usando, para tanto, os pontos mais centrais das escalas. Esse aparente autofavoritismo dos empregados (também chamado de efeito de leniência) é recorrente em estudos que comparam autoavaliação de desempenho com avaliações realizadas por supervisores (para mais informações ver Golman \& Bhatia, 2012).

No que diz respeito às correlações com as variáveis positivas, os resultados de esperança e desempenho autoavaliado vão ao encontro de outros estudos (Luthans et al., 2007; Peterson \& Byron, 2008). Os resultados do estudo de Peterson e Byron (2008), por exemplo, sugerem que escores mais altos em esperança tendem a estar relacionados a um leque maior e com mais qualidade de soluções para os problemas propiciando melhor desempenho. Pode-se pensar que a combinação do mecanismo de agenciamento presente na esperança e a capacidade de criar rotas seja, em grande parte, responsável por um desempenho superior. Assim, por sentirem-se mais motivados para cumprir metas de trabalho, profissionais com alta esperança teriam condi- 
ções de gerar várias estratégias para atingir um objetivo determinado.

Diferentemente, a esperança e o desempenho avaliado pelo supervisor não apresentaram correlação. Esse resultado se assemelha ao obtido por Simmons, Gooty, Nelson e Little (2009). Segundo esses autores, uma possível explicação para a ausência de uma relação significativa entre a esperança e o desempenho avaliado pelo superior pode residir no fato de subordinados e supervisores estarem focando em metas diferentes quando avaliam o desempenho (Simmons et al., 2009). Por exemplo, um profissional da área de vendas que atinge a meta de vendas mensal mínima estabelecida pode avaliar seu desempenho positivamente, enquanto seu supervisor, ao comparar o desempenho dos membros da equipe, avalia que esse resultado é regular ou mesmo insuficiente.

Com relação aos resultados de otimismo e desempenho, verificaram-se associações positivas, como também encontraram Kluemper, Little e DeGroot (2008). Seligman (1998) destaca que ser otimista faz a diferença na realização de um trabalho bem feito, mal feito ou não feito, sugerindo que os mais otimistas apresentam resultados de maior qualidade. Isso porque as pessoas otimistas tendem a tornar-se mais focadas e acreditam que podem alcançar seus objetivos, apesar das dificuldades. Além disso, como defendem Avey et al. (2009) indivíduos mais otimistas apresentam maior motivação e empregam mais esforços para realizar tarefas, o que poderia acarretar em melhor desempenho profissional.

A ausência de correlação entre o otimismo e o desempenho avaliado pelo supervisor pode ser explicada por uma tendência dos subordinados de ter uma percepção excessivamente positiva sobre seu desempenho que não corresponde à percepção do supervisor. Segundo Lovallo e Kahneman (2003), os seres humanos apresentam uma poderosa tendência a exagerar seus próprios talentos, a acreditar que são superiores à média no que diz respeito a seus traços positivos e habilidades. Essa tendência provavelmente faz com que os profissionais percebam a qualidade do trabalho realizado com o mesmo exagero. Os supervisores, em contrapartida, adotariam o que Lovallo e Kahneman (2003) chamam de visão externa. Essa visão seria significativamente mais objetiva e confiável do que a visão interna (autoavaliação). Em suma, é possível que a ausência de correlação entre otimismo e o desempenho avaliado pelo supervisor, assim como ocorre com a esperança, se deva a uma discrepância entre as avaliações feitas por supervisores e subordinados.

No que se refere às relações de desempenho autoavaliado e as dimensões de criatividade Integração e analogia e Soluções e efeitos, encontraram-se correlações positivas, tal como evidenciaram outros autores (Davidovitz \& Milgram, 2006; Mäkikangas \& Kinnunem, 2003; Wechsler, 2006). O mesmo não ocorreu com o desempenho avaliado pelos supervisores. É interessante notar que a dimensão Soluções e efeitos, que diz respeito à ponderação sobre elementos externos às ideias e às soluções cogitadas apresentou uma correlação positiva e moderada com o desempenho autoavaliado e uma correlação também positiva, porém fraca, com o desempenho avaliado pelos supervisores. Diferentemente, a dimensão integração e analogia, que diz respeito à capacidade de associar perspectivas e apresentar diversificação de ideias não teve correlação com o desempenho avaliado pelos supervisores. Esses resultados, combinados, sugerem que para os supervisores é importante, em alguma medida, que seus subordinados sejam capazes não de ter ideias diversificadas, mas sim de considerar a realidade externa, as consequências, a exequibilidade de suas ideias, e que sejam capazes de comunicar aquilo que pensam de forma clara.

Além dos testes de correlações com os escores de desempenho geral, testaram-se modelos explicativos do desempenho incluindo-se como explicativas as variáveis relacionadas. No modelo preditivo de desempenho autoavaliado, foram variáveis explicativas significativas: a esperança e o fator de criatividade soluções e efeitos, ambas preditoras positivas. Observou-se que uma razoável parcela da variância do desempenho foi explicada por essas variáveis (43\%), inclusive o 
valor do $R^{2}$ ajustado foi semelhante. No que diz respeito à esperança, esse resultado vai ao encontro de estudos realizados em outros países (Avey et al., 2009; Luthans et al., 2007; Wright \& Quick, 2009). Chama a atenção no modelo a saída da variável otimismo e a permanência da criatividade. Tais resultados sugerem que quando essas variáveis positivas são tomadas em conjunto, as que guardam poder explicativos sobre o desempenho são aquelas relacionadas à percepção de metas e estratégias criativas relacionadas às tarefas. Considerando a natureza dos cargos ocupados pelos participantes (vendedores e recuperadores de crédito) acredita-se que o resultado vai ao encontro do que é esperado no exercício de suas funções, uma vez que em ambas, com frequência, é preciso lançar mão de ideias criativas - ou não tradicionais - para executar uma venda ou encontrar uma solução para que os clientes quitem suas dívidas.

Com relação aos modelos preditivos do desempenho avaliado pelos supervisores, as variáveis significativas foram o mesmo fator da criatividade, Soluções e efeito, e a idade de modo negativo. Apesar de o modelo ter sido significativo, ressalta-se que ele explicou pouco da variância do desempenho avaliado pelos supervisores ( $9 \%$ ), e o valor ajustado do $R^{2}$ mostrou-se ainda menor. Pode-se relacionar o resultado da criatividade ao fato de os participantes da pesquisa encontrarem-se em atividades em que possuíam pouco tempo para elaborar planos de ação, portanto, é possível que aqueles mais altos nesse fator de criatividade tenham sido percebidos como desempenhando melhor sua função. Sobre as atividades de trabalho desempenhadas pelos participantes, é importante notar que em muitas situações, o cliente estaria presente, seja fisicamente, on-line ou pelo telefone, esperando uma resposta. Por essa razão, os funcionários precisavam solucionar rapidamente a demanda do cliente e, ao mesmo tempo, pensar no impacto de cada ação tomada, a fim de deixar a organização e os clientes satisfeitos. No que se refere à idade, pode-se pensar que a agilidade associada ao estereótipo de jovens incida sobre a percepção de desempenho dos supervisores.

De maneira geral, os resultados desse estudo sugerem que a discrepância entre as avaliações de desempenho feitas pelos profissionais e por seus supervisores possa estar relacionada à falta de clareza na comunicação, especialmente no que se refere às expectativas individuais e objetivos organizacionais. Essa possibilidade fortalece a noção de que a relação entre as características individuais positivas e os resultados valorizados no trabalho será mais forte quando subordinados e seus supervisores forem capazes de estabelecer um diálogo significativo, porém desafiador sobre o que é esperado no trabalho.

Considera-se que este trabalho ofereceu importantes contribuições para o desenvolvimento teórico da Psicologia Organizacional Positiva ao apresentar dados empíricos que replicam achados internacionais em um contexto brasileiro. Estudos dessa natureza ainda são escassos fora da América do Norte, e especialmente nas Américas Central e do Sul, e os resultados encontrados salientam a importância de novas investigações sobre o tema. Mais especificamente, os achados deste estudo destacam evidências sobre como a esperança, o otimismo e a criatividade estão relacionados com o desempenho profissional. Tais resultados são especialmente importantes ao se considerar que as características positivas investigadas são passíveis de desenvolvimento. Isso significa dizer que programas educacionais e de desenvolvimento de pessoas podem, além de contribuir para o próprio florescimento pessoal dos indivíduos, fomentar o desempenho de organizações.

\section{Referências}

Aguinis, H., Gottfredson, R. K., \& Joo, H. (2012). Using performance management to win the talent war. Business Horizons, 55, 609-616. doi:10.1016/j.bushor.2012.05.007

Amabile, T. M. (1993). What does a theory of creativity require? Psychological Inquiry, 4(2), 179-181. doi:10.1207/s15327965pli0403 2

Amabile, T. M. (1998). How to kill creativity. Harvard Business Review, 76(5), 76-87.

Amabile, T. M., \& Gryskiewicz, S. S. (1988). Creative human resources in the R\&D laboratory: How environment and personality impact inno- 
vation. In R. L. Kuhn (Ed.), Handbook for creative and innovative managers (pp. 501-524). New York: McGraw-Hill.

Avey, J. B., Luthans, F., \& Youssef, C. M. (2009). The additive value of positive psychological capital in predicting work attitudes and behaviors. Journal of Management, 36, 430-452. doi:10.1177/0149206308329961

Bastianello, M. R., Zanon, C., Pacico, J. C., \& Hutz, C. S. (no prelo). Optimism, self-esteem, and personality: Adaptation and validation of the Brazilian version of the LOT-R. Psico-UFS.

Carver, C. S., \& Scheier, M. F. (2005). Optimism. In C. R. Snyder \& S. J. Lopez (Eds.), The handbook of positive psychology (pp. 231-243). New York: Oxford University Press.

Csikszentmihalyi, M. (1996). Creativity. New York: Harper Collins.

Davidovitz, N., \& Milgram, R. M. (2006). Creative thinking as a predictor of teacher effectiveness in higher education. Creativity Research Journal, 18, 385-390. doi:10.1207/s15326934crj1803_12

Donaldson, S. I., \& Ko, I. (2010). Positive organizational psychology, behavior, and scholarship: A review of the emerging literature and evidence base. The journal of Positive Psychology, 5(3), 177-191. doi:10.1080/17439761003790930

Golman, R., \& Bhatia, S. (2012). Performance evaluation inflation and compression. Accounting, Organizations and Society, 37(8), 534-543. doi:10.1016/j.aos.2012.09.001

Holmes, J. (2007). Making humor work: Creativity on the job. Oxford University Press, 28(4), 518537.

Kluemper, D. H., Little, L. M., \& DeGroot, T. (2008). State or trait: Effects of state optimism on jobrelated outcomes. Journal of Organizational Behavior, 30, 209-231. doi:10.1002/job.591

Lewis, R. E., \& Heckman, R. J. (2006). Talent management: A critical review. Human Resource Management Review, 16, 139-154. doi:10.1016/j.hrmr.2006.03.001

Lovallo, D., \& Kahneman, D. (2003). Delusions of success: How optimism undermines executive's decisions. Harvard Business Review, 81(7), 56-63.

Luthans, F. (2002). The need for and meaning of positive organizational behavior. Journal of Organizational Behavior, 23, 695-706. doi:10.1002/ job. 165
Luthans, F., Avolio, B. J., Avey, J. B., \& Norman, S. M. (2007). Positive psychological capital: Measurement and relationship with performance and satisfaction. Personnel Psychology, 60, 541572. doi:10.1111/j.1744-6570.2007.00083.x

Luthans, F., \& Youssef, C. M. (2004). Human, social, and now positive psychological capital management: Investing in people for competitive advantage. Organizational Dynamics, 33(2), 143-160.

Mäkikangas, A., \& Kinnunen, U. (2003). Psychosocial work stressors and well-being: Self-esteem and optimism as moderators in a one-year longitudinal sample. Personality and Individual Differences, 35, 537-557. doi:10.1016/S01918869(02)00217-9

Martin, A. J. (2005). The role of positive psychology in enhancing satisfaction, motivation, and productivity in the workplace. Journal of Organizational Behavior Management, 24, 113-133.

Moraes, M. M., \& Lima, S. M. V. (2009). Estratégias para criar no trabalho: Proposição teórica e validação psicométrica de medida. Paidéia (Ribeirão Preto), 44(19), 367-377.

Montag, T., Maertz, C. P., Jr., \& Baer, M. (2012). A critical analysis of the workplace creativity criterion space. Journal of Management, 38(4), 1362-1386. doi:10.1177/0149206312441835

Pacico, J. C., Zanon, C., Bastianello, M. R., \& Hutz, C. S. (2013). Adaptation and validation of the Brazilian version of the Hope Index. International Journal of Testing, 13, 193-200.

Peterson, S. J., \& Byron, K. (2008). Exploring the role of hope in job performance: Results from four studies. Journal of Organizational Behavior, 29, 785-803. doi:10.1002/job.492

Seligman, M. E. P. (1998). Learned optimism. New York: Pocket Books.

Simmons, B. L., Gooty, J., Nelson, D., \& Little, L. M. (2009). Secure attachement: Implications for hope, trust, burnout and performance. Journal of Organizational Behavior, 30, 233-247. doi:10.1002/job.585

Siqueira, W. (2002). Avaliação de desempenho: Como romper amarras e superar modelos ultrapassados. Rio de Janeiro, RJ: Reichmann \& Affonso Editores.

Snyder, C. R., \& Lopez, S. J. (2005). The handbook of positive psychology. New York: Oxford University Press.

Tierney, P., Farmer, S. M., \& Graen, G. B. (1999). 
An examination of leadership and employee creativity: The relevance of traits and relations. Personnel Psychology, 52, 591-620. doi:10.1111/j.1744-6570.1999.tb00173.x

Turner, N., Barling, J., \& Zacharatos, A. (2002). Positive psychology at work. In C. R. Snyder \& S. J. Lopez (Eds.), Handbook of positive psychology (pp. 715-728). New York: Oxford University Press.

Vazquez, A. C., \& Hutz, C. S. (2008). Escala Vazquez-Hutz de avaliação de desempenho. São Paulo, SP: Casa do Psicólogo.

Youssef, C. M., \& Luthans, F. (2007). Positive organizational behavior in the workplace: The impact of hope, optimism, and resilience. Journal of Management, 33(5), 740-880. doi:10.1177/0149206307305562

Wechsler, S. M. (2006). Estilos de pensar e criar. Campinas, SP: Laboratório de Avaliação e Medidas Psicológicas, Pontifícia Universidade $\mathrm{Ca}$ tólica de Campinas.
Wiegand, D. M., \& Geller, E. S. (2005). Connecting positive psychology and organizational behavior management: Achievement motivation and the power of positive reinforcement. Journal of Organizational Behavior Management, 24, 3-25. doi:10.1300/J075v24n01_02

Wright, T. A., \& Quick, J. C. (2009). The role of positive-based research in building the science of organizational behavior. Journal of Organizational Behavior, 30, 329-336. doi:10.1002/job.581

Recebido: 05/12/2013

$1^{a}$ revisão:24/02/2014

Aceite final: 27/02/2014 